\title{
PDGF-AA and bFGF mediate B104CM-induced proliferation of oligodendrocyte precursor cells
}

\author{
JIAN-GUO HU ${ }^{1,3^{*}}$, XING-JUN WU ${ }^{2 *}$, YI-FAN FENG ${ }^{1,3}$, GANG-MING XI ${ }^{2}$, \\ ZHEN-HUAN WANG ${ }^{3}$, JIAN-SHENG ZHOU ${ }^{1}$ and HE-ZUO L $\ddot{U}^{1,3}$
}

\author{
${ }^{1}$ Department of Clinical Laboratory Science, The First Affiliated Hospital of Bengbu Medical College, \\ Bengbu, Anhui 233004; ${ }^{2}$ Department of Neurology, Xuhui Central Hospital, Shanghai 200031; ${ }^{3}$ Anhui Key \\ Laboratory of Tissue Transplantation, Bengbu Medical College, Bengbu, Anhui 233004, P.R. China
}

Received May 31, 2012; Accepted August 6, 2012

DOI: $10.3892 /$ ijmm.2012.1110

\begin{abstract}
The conditioned medium from B104 neuroblastoma cells (B104CM) induces proliferation of oligodendrocyte precursor cells (OPCs) in vitro, which indicates that certain factors contained within B104CM give instructional signals that direct the proliferation of OPCs. However, the OPC-proliferative factors present in B104CM have yet to be identified. Platelet-derived growth factor AA (PDGF-AA), basic fibroblast growth factor (bFGF) and insulin-like growth factor-1 (IGF-1) have been reported to act as potent mitogens for OPC proliferation. This raises the possibility that B104CM induces proliferation of OPCs through secretion of PDGF-AA, bFGF and/or IGF-1. In the present study, we detected the expression and levels of PDGF-AA, bFGF and IGF-1 in B104 cells and $\mathrm{B} 104 \mathrm{CM}$, and observed the expression of their receptors in OPCs. The results indicated that these growth factors were expressed in B104 cells and B104CM. All 3 receptors, PDGFR, FGFR2 and IGF-1R, were also detected in OPCs. Furthermore, B104CM-stimulated OPC proliferation could be markedly decreased by both AG1295 (an inhibitor of PDGFR) and PD173074 (an inhibitor of FGFR). However, the inhibition of IGF-1R with AG1204 did not affect the proliferation of OPCs. Our study suggests that the PDGF-AA and bFGF in $\mathrm{B} 104 \mathrm{CM}$ are 2 key factors that stimulate OPC proliferation.
\end{abstract}

\section{Introduction}

Oligodendrocyte precursor cells (OPCs) play an important role not only as precursor cells that give rise to myelinating cells in the central nervous system (CNS), but also as active

Correspondence to: Professor He-Zuo Lü, Anhui Key Laboratory of Tissue Transplantation, Bengbu Medical College, 287 Chang Huai Road, Bengbu, Anhui 233004, P.R. China

E-mail: jghu.hzlv@yahoo.com

${ }^{*}$ Contributed equally

Key words: B104 neuroblastoma cells, oligodendrocyte precursor cells, proliferation, platelet-derived growth factor AA, basic fibroblast growth factor participants in the neural network. Generation of OPCs from multi-potent neural stem cells (NSCs) is considered to be induced by factors produced by the notochord and/or floor plate of the neural tube (1). These OPCs express specific markers A2B5, NG2 and PDGFR $\alpha$ and can differentiate into oligodendrocytes in serum-free medium or type-2 astrocytes in serum-containing medium (2).

The B104 neuroblastoma cell is a neuronal cell line. It is well known that the conditioned medium prepared from B104 neuroblastoma cells (B104CM) expands the OPCs in vitro (3). However, the OPC-proliferative factors present in B104CM have yet to be identified.

It has been reported the purified OPCs from postnatal rat optic nerve, cultured in serum-free medium containing platelet-derived growth factor AA (PDGF-AA) and basic fibroblast growth factor (bFGF), are able to undergo continuous self-renewal in the absence of differentiation $(4,5)$. Moreover, a previous study showed that insulin-like growth factor-1 (IGF-1) also induced OPC proliferation in vitro (6). However, whether PDGF-AA, bFGF and/or IGF-1 are key factors in B104CMinduced OPC proliferation remains unknown. Thus, in the present study, we examined the expressions of PDGF-AA, bFGF and IGF-1 in the B104 cell line and observed the effects of their inhibitors on B104CM-induced OPC proliferation.

\section{Materials and methods}

Isolation and culture of spinal cord-derived OPCs. OPCs were immunopanned from embryonic day (E) 15 Wistar rat spinal cords using an A2B5 antibody and a protocol modified from previous studies (7-9). Immunopanned OPCs were plated onto PDL/fibronectin-coated 10x12 cm culture dishes and growth medium was added and changed every other day. The growth medium contained DMEM/Ham's F12, 1xN2 and 1xB27 supplements, fibroblast growth factor 2 (FGF-2, $20 \mathrm{ng} / \mathrm{ml}$ ) (Invitrogen, Carlsbad, CA, USA), and PDGF-AA, (10 ng/ml; R\&D Systems, Minneapolis, MN, USA). In all cases, an aliquot of cells was analyzed the following day to determine the efficiency of immunopanning. Only preparations in which $>90 \%$ of the bound cells expressed A2B5 were used. After 5-7 days, the cells were passaged. In all experiments, cells at passage 2 (P2) were used. 
Table I. Sequences of primers and PCR product sizes used in RT-PCR.

\begin{tabular}{|c|c|c|c|}
\hline Genes & & Primer sequences & Size (bp) \\
\hline PDGF-AA & $\begin{array}{l}\text { Sense } \\
\text { Antisense }\end{array}$ & $\begin{array}{l}\text { 5'-TGTGCCCATCCGCAGGAAGAG-3' } \\
\text { 5'-TTGGCCACCTTGACACTGCG-3' }\end{array}$ & 225 \\
\hline $\mathrm{bFGF}$ & $\begin{array}{l}\text { Sense } \\
\text { Antisense }\end{array}$ & $\begin{array}{l}\text { 5'-GGCTTCTTCCTGCGCATCCA-3' } \\
\text { 5'-GCTCTTAGCAGACATTGGAAGA-3' }\end{array}$ & 353 \\
\hline IGF-1 & $\begin{array}{l}\text { Sense } \\
\text { Antisense }\end{array}$ & $\begin{array}{l}\text { 5'-GGGCA TTGTGGATGAGTG-3' } \\
\text { 5'-CAAAGGATCTTGCGGTGA-3' }\end{array}$ & 246 \\
\hline$\beta$-actin & $\begin{array}{l}\text { Sense } \\
\text { Antisense }\end{array}$ & $\begin{array}{l}\text { 5'-ATTGTAACCAACTGGGACG-3' } \\
\text { 5'-TTGCCGATAGTGATGACCT-3' }\end{array}$ & 533 \\
\hline
\end{tabular}

Animals. All embryonic rats were obtained from female pregnant SD rats bred in the Animal Care Facility at Bengbu Medical College. All animal care was carried out in accordance with the National Institute of Health Guide for the Care and Use of Laboratory Animals (NIH publication no. 80-23; revised 1996) and was approved by the Bengbu Medical College Animal Care Committee of the Use of Laboratory Animals.

Preparation of B104CM. B104 neuroblastoma cells were a generous gift from Dr Ian Duncan (University of Wisconsin) and $\mathrm{B} 104 \mathrm{CM}$ was prepared according to the method of Louis et al (3). Cultures of B104 neuroblastoma cells were maintained in logarithmic phase of growth in DMEM (Invitrogen) supplemented with $10 \%$ FCS. For conditioned medium (B104CM) production, cultures $\left(100-150\right.$ cells $\left./ \mathrm{mm}^{2}\right)$ were washed 2 times with Hank's salt solution and incubated in serum-free DMEM. Three days later, the medium was removed, filtered (Nalgene filters, $0.45 \mu \mathrm{m}$ ) and stored at $-20^{\circ} \mathrm{C}$.

Immunocytochemistry. OPCs were plated onto PDL/ fibronectin-coated coverslips in $35-\mathrm{mm}$ dishes at a density of $5 \times 10^{4}$ cells/coverslip (12 mm). Oligodendrocytes (OLs) and type- 2 astrocytes were induced from OPCs as previously described (10). To identify the purity of OPCs, to detect the expression of several receptors in OPCs and to confirm the differentiation potential of OPCs, OPCs as well as the induced OLs and type- 2 astrocytes cultured on coverslips were rinsed in PBS and fixed with $4 \%$ paraformaldehyde (PFA) in PBS for $20 \mathrm{~min}$ at room temperature (RT). After 3 rinses in PBS (10 min each), the cells were incubated with $10 \%$ normal goat serum (NGS) in PBS in the presence (for intracellular antigens) or absence (for surface markers) of $0.3 \%$ Triton X-100 for $1 \mathrm{~h}$ at RT and then with one of the monoclonal primary antibodies against A2B5 (1:100), and NG2 (1:100), the markers for OPCs, Rip (1:100) (Millipore, Billerica, MA, USA), the markers for oligodendrocytes, glial fibrillary acidic protein (GFAP; 1:200; Sigma-Aldrich, St. Louis, MO, USA), the marker for type-2 astrocytes, PDGFR (1:500; Abcam, Cambridge, MA, USA), FGFR2 (1:500; Abcam) or IGF-1R (1:100; Millipore) overnight at $4{ }^{\circ} \mathrm{C}$. On the second day, the cultures were incubated with rhodamine- or fluorescein isothiocyanate (FITC)-conjugated secondary antibody (Jackson ImmunoResearch Laboratories, Inc., West Grove, PA, USA) for $1 \mathrm{~h}$ at $37^{\circ} \mathrm{C}$. After staining, the coverslips were rinsed and mounted with Gel/Mount aqueous mounting media (Biomeda Corp., Foster City, CA, USA) containing Hoechst 33342, a fluorescent nuclear dye $(1 \mu \mathrm{g} /$ ml;Sigma-Aldrich). The coverslips were examined using an Olympus BX60 microscope. For cell counts, at least 5 randomly selected fields with a total of $>500$ cells were counted. In all experiments, primary antibody omission controls were used to confirm the specificity of the immunofluorescence labeling.

5-bromo-2'-deoxyuridine (BrdU) incorporation assay. To assess proliferation of OPCs cultured under B104CM, we used in situ BrdU incorporation assay. Briefly, the cultured OPCs at passage 2 were seeded onto poly-L-lysine $(200 \mu \mathrm{g} / \mathrm{ml})$-coated coverslips at a density of $3 \times 10^{4}$ cells/coverslip and cultured in growth medium with FGF and PDGF-AA. After $24 \mathrm{~h}$, both PDGF and bFGF were withdrawn from the OPC-medium and the cells were cultured another $24 \mathrm{~h}$. Then, different concentrations of $\operatorname{B104CM}(0,10,30,50$ and $100 \%)$ were added into the culture medium. The OPCs were allowed to grow for $24 \mathrm{~h}, \mathrm{BrdU}$ (10 $\mu \mathrm{M}$; Sigma-Aldrich) was added to the medium for $16 \mathrm{~h}$, and cells were fixed in 4\% PFA for 15 min and washed twice with PBS. For anti-BrdU and A2B5 immunofluorescence doublelabeling, the cells were treated with $1 \mathrm{~N} \mathrm{HCl}$ for 40 min at $37^{\circ} \mathrm{C}$ to denature the DNA prior to the use of primary (mouse antiBrdU; 1:80; Dako, Santa Barbara, CA, USA; mouse anti-A2B5 $\operatorname{IgM} \mathrm{mAb}$ ) and secondary antibody (rhodamine-conjugated goat anti-mouse IgG, 1:50, Sigma-Aldrich; FITC-conjugated donkey anti-mouse IgM, 1:200, Jackson ImmunoResearch Lab, Inc.). The coverslips were examined and photographed using an Olympus BX60 microscope.

Reverse transcription-polymerase chain reaction (RT-PCR). RT-PCR was used to detect the expression of PDGF-AA mRNA in B104 cells. Briefly, total-RNA from B104 cells was extracted with the TRIzol (Invitrogen) according to the manufacturer's instructions. Two micrograms of total-RNA was first reverse transcribed into cDNA, and then PCR was performed by a routine method (11). PCR products were analyzed on $1 \%$ agarose gel. $\beta$-actin was used as an internal control. The sequences of specific primers for RT-PCR are given in Table I.

Measurement of PDGF-AA, bFGF and IGF-1 proteins in B104CM by enzyme-linked immunosorbent assay (ELISA). The levels of PDGF-AA, bFGF and IGF-1 proteins in B104CM 


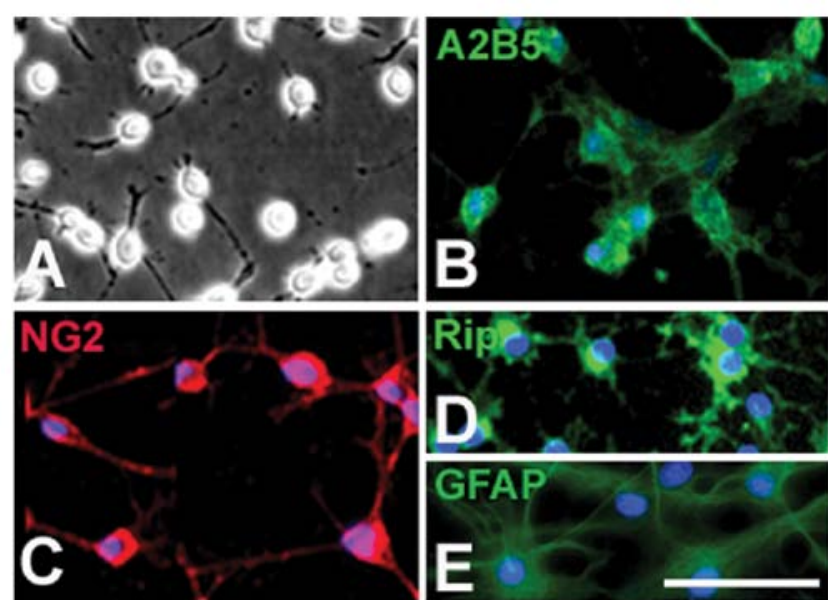

Figure 1. Morphological and immunocytochemical characteristics of OPCs. (A) Phase contrast photomicrograph of OPCs cultured in growth medium supplemented with $20 \mathrm{ng} / \mathrm{ml}$ PDGF-AA and $20 \mathrm{ng} / \mathrm{ml}$ bFGF. (B) The cells were immunopositive for A2B5 (green). (C) The cells were immunopositive for NG2 (red). (D) When cultured in growth factor-free medium for 5 days, OPCs differentiated into Rip positive oligodendrocytes (green). (E) When cultured in growth factor-free and 10\% FBS contained growth medium for 5 days, OPCs differentiated into GFAP positive type II astrocytes (green). (B-E) Cells were counterstained with Hoechst 33342 (blue), a nuclear dye. Scale bar, $25 \mu \mathrm{m}$

were quantified using commercially available ELISA kits (R\&D Systems). ELISA assay was performed according to the manufacturer's instructions. Briefly, assay diluents (100 $\mu \mathrm{l})$ were added to each well that had been pre-coated with a monoclonal antibody specific for PDGF-AA, bFGF or IGF-1. This was followed by the addition of $50 \mu 1$ of control standard or sample/well and incubation for $2 \mathrm{~h}$ at RT on a horizontal orbital microplate shaker at $500 \mathrm{rpm}$. Each well was aspirated and subsequently washed with wash buffer using an autowasher; this was repeated 3 times for a total of 4 washes. After removing any remaining wash buffer, $200 \mu 1$ of PDGF-AA, bFGF or IGF-1 conjugate was added to each well and plates were incubated for $2 \mathrm{~h}$ at RT on a shaker. Aspiration and washes were repeated as described above. Substrate solution ( $200 \mu 1)$ was added to each well and the plates were incubated for $30 \mathrm{~min}$ at RT, protected from light. Stop solution $(50 \mu 1)$ was added to each well. The optical density of each well was determined using a microplate reader at $450 \mathrm{~nm}$. PDGF-AA, bFGF or IGF-1 concentrations of samples were determined from the optical densities in relation to standard experimental curves. No interference and no cross reactivity was expected based on the manufacturer's instructions. Each sample was measured three times and the mean level of each measurement was used for analysis.

Treatment of OPCs with the inhibitors of growth factor signals. To identify the roles of PDGF-AA, bFGF and IGF-1 in B104CM-induced OPC proliferation, the OPC cultures were pretreated with AG1295 (a specific inhibitor of the PDGFR signal pathway; $5 \mu \mathrm{M}$; R\&D Systems), PD173074 (a specific inhibitor of the bFGFR signal pathway; $2 \mu \mathrm{M}$; Sigma-Aldrich) or AG1204 (a specific inhibitor of the IGFR signal pathway; $5 \mu \mathrm{M}$; Calbiochem, La Jolla, CA, USA) for $45 \mathrm{~min}$ before adding of B104CM-contained OPC-growth medium each time.

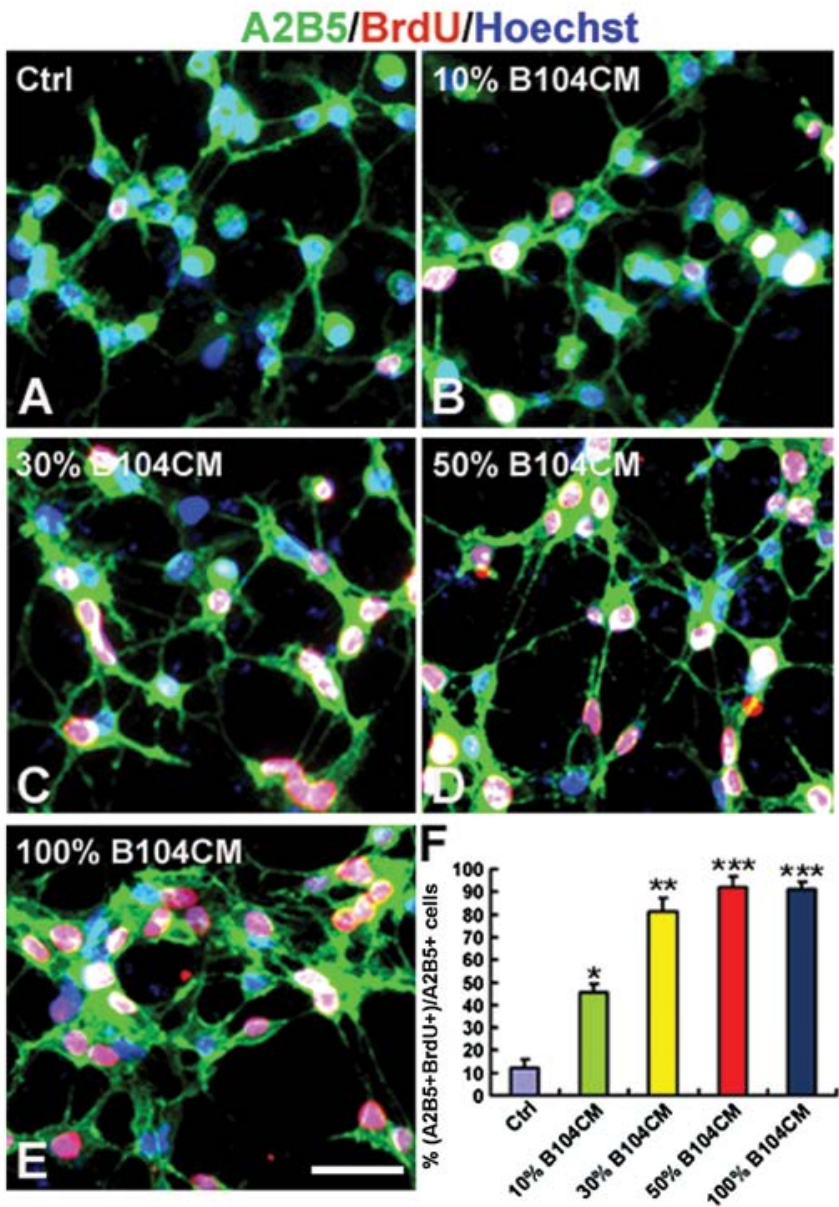

Figure 2. B104CM promotes proliferation of cultured OPCs in vitro. (A-E) Representative photomicrographs displayed BrdU-positive OPCs when cells were cultured for 3 days in (A) control, (B) $10 \%$, (C) $30 \%$, (D) $50 \%$ and (E) $100 \%$ B104CM. Cells were incubated with $\operatorname{BrdU}(10 \mu \mathrm{M})$ for $16 \mathrm{~h}$ before cell fixation. Immunofluorescent double labeling of BrdU (red) and A2B5 (green) was performed and all nuclei were stained with Hoechst 33342 (blue). (F) Quantitative measures demonstrate that treatment of OPCs with $\mathrm{B} 104 \mathrm{CM}$ resulted in a significant increase of $\mathrm{BrdU}^{+}$cells in $\mathrm{A} 2 \mathrm{~B}^{+} \mathrm{OPCs}$. Data presented are mean $\pm \mathrm{SD}(\mathrm{n}=5) .{ }^{*} \mathrm{P}<0.05,{ }^{* *} \mathrm{P}<0.01,{ }^{* * * *} \mathrm{P}<0.001$, compared to the control. Scale bar, $25 \mu \mathrm{m}$.

Statistical analysis. Data are presented as the mean \pm standard deviation of the mean (SD). One-way analysis of variance (ANOVA) with post hoc Tukey's t-test was used to determine statistical significance. P-value $<0.05$ was considered to indicate statistically significant differences.

\section{Results}

Cultivation and identification of OPCs. The OPCs displayed typical bipolar or tri-polar morphology (Fig. 1A) and expressed A2B5 (Fig. 1B) and PDGFR (Fig. 1C), the markers of OPCs. When cultured in the differentiation medium (without PDGF-AA and bFGF) for 5 days, these cells displayed a multi-polar morphology and the majority expressed oligodendrocyte-specific marker RIP (Fig. 1D). However, when OPCs were cultured in the presence of $10 \%$ FBS, almost all displayed the typical process-bearing morphology of astrocytes and expressed GFAP (Fig. 1E). 

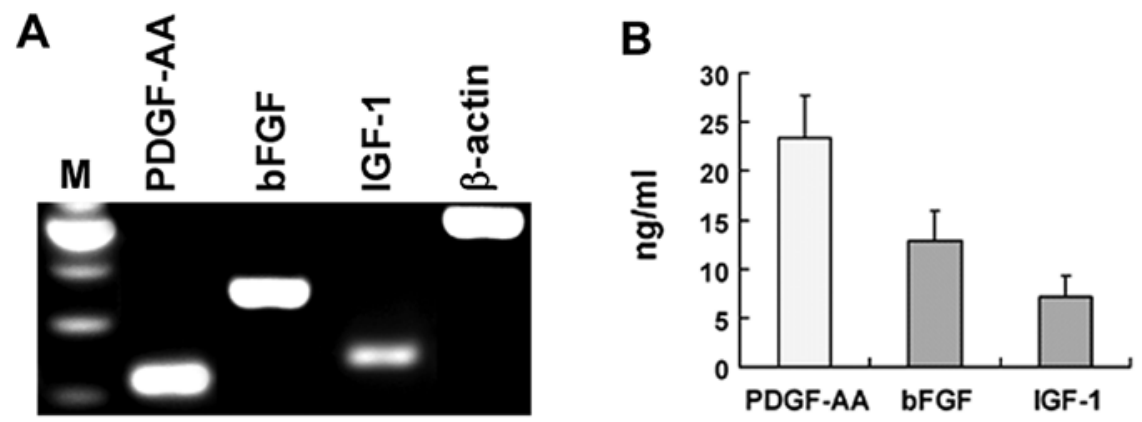

Figure 3. mRNA expressions of PDGF-AA, bFGF and IGF-I in the B104 cell line and their concentrations in protein level within B104CM. (A) RT-PCR analysis of the mRNA expressions of PDGF-AA, bFGF and IGF-I in the B104 cell line. (B) The concentrations of PDGF-AA, bFGF and IGF-I in B104CM were determined by ELISA.
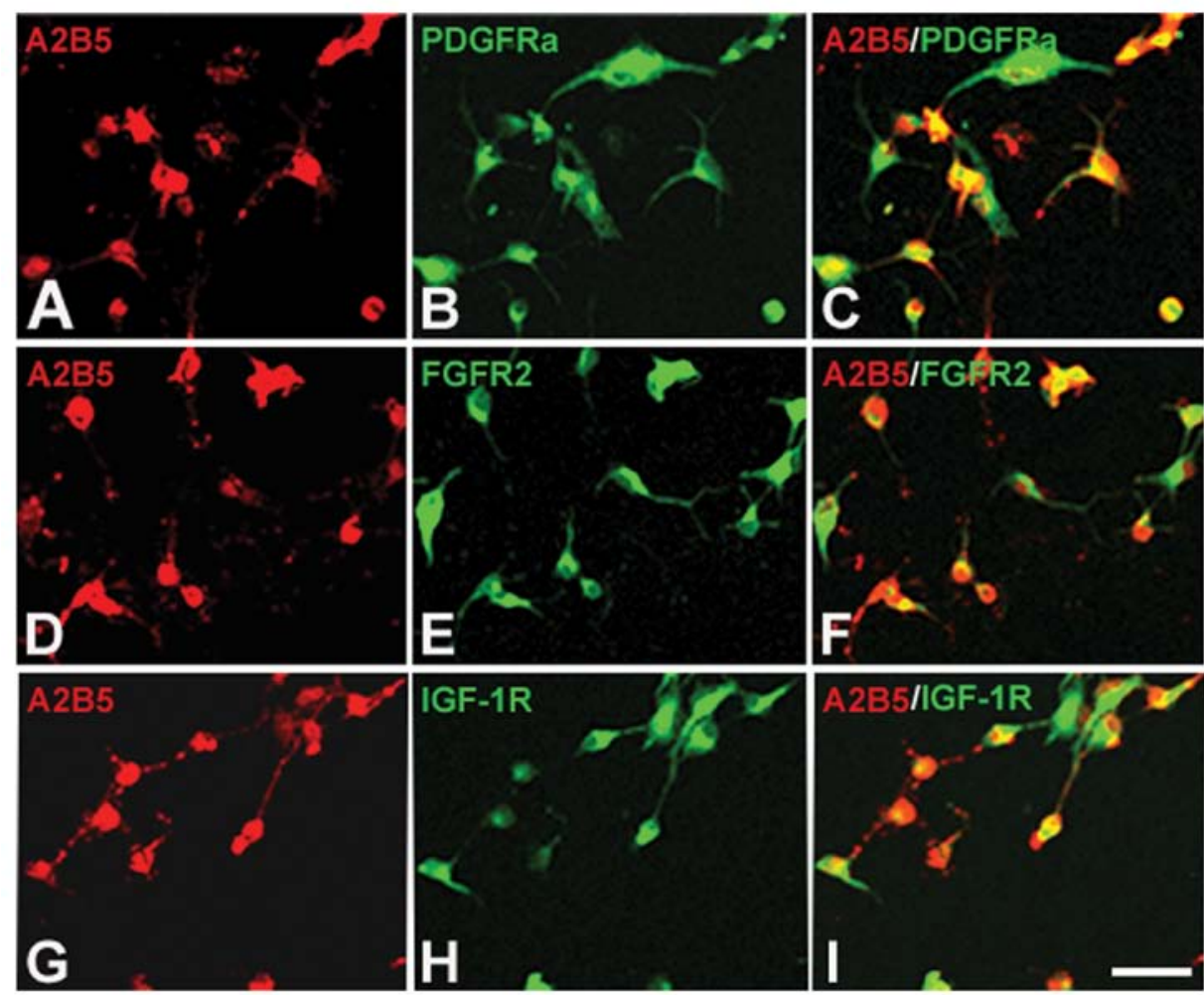

Figure 4. Expression of PDGFRa, FGFR2 and IGF-1R in OPCs. The expressions of (A-C) PDGFR $\alpha$, (D-F) FGFR2 and (G-I) IGF-1R in OPCs were confirmed by immunofluorescence staining. All cell nuclei were labeled with Hoechst 33342 (blue). Scale bar, $50 \mu \mathrm{m}$.

Proliferation of OPCs induced by B104CM. The effect of B104CM on the proliferation of OPCs was determined using $\mathrm{BrdU}$ incorporation assay. We counted the number of BrdUpositive OPCs cultured in the control medium and different concentrations of B104CM (Fig. 2A-F). The result showed that the percentage of BrdU-positive cells was significantly increased in A2B5-positive OPCs cultured in B104CM of $10 \%(45.28 \pm 3.91 \%, \mathrm{P}<0.05), 30 \%(81.45 \pm 5.69 \%, \mathrm{P}<0.01)$ and $50 \%(91.92 \pm 4.52 \%, \mathrm{P}<0.001)$ and $100 \%(91.78 \pm 3.22 \%$, $\mathrm{P}<0.001)$ compared to the control group $(12.24 \pm 3.76 \%)$. The proliferation of OPCs reached peak values when cultured in 50\% B104CM (Fig. 2D and F). This result strongly suggests that $\mathrm{B} 104 \mathrm{CM}$ is a strong promoter of OPC proliferation.

mRNA expression and protein concentrations of PDGF-AA, bFGF and IGF-1 in B104 cells and B104CM, respectively.
To determine the possibility that PDGF-AA, bFGF and IGF-1 mediate B104CM-induced proliferation of OPC, we firstly confirmed whether B104 cells express these 3 growth factors. Using RT-PCR, we showed that the mRNA of PDGFAA, bFGF and IGF-1 was expressed substantially in B104 cells (Fig. 3A). More importantly, we detected these growth factors in protein level in B104CM by ELISA. The concentration of PDGF-AA, bFGF and IGF-1 in B104CM (without being concentrated) reached $23.42 \pm 4.28,12.94 \pm 3.05$ and $7.21 \pm 2.12 \mathrm{ng} / \mathrm{ml}$, respectively (Fig. 3B).

Expression of growth factor receptors in OPCs. The functions of growth factors rely on binding to their receptors. If they can induce OPCs to proliferate, the OPCs should express their receptors. To determine whether the OPCs cultured in our system express the receptors of PDGF-AA, bFGF and IGF-1, 

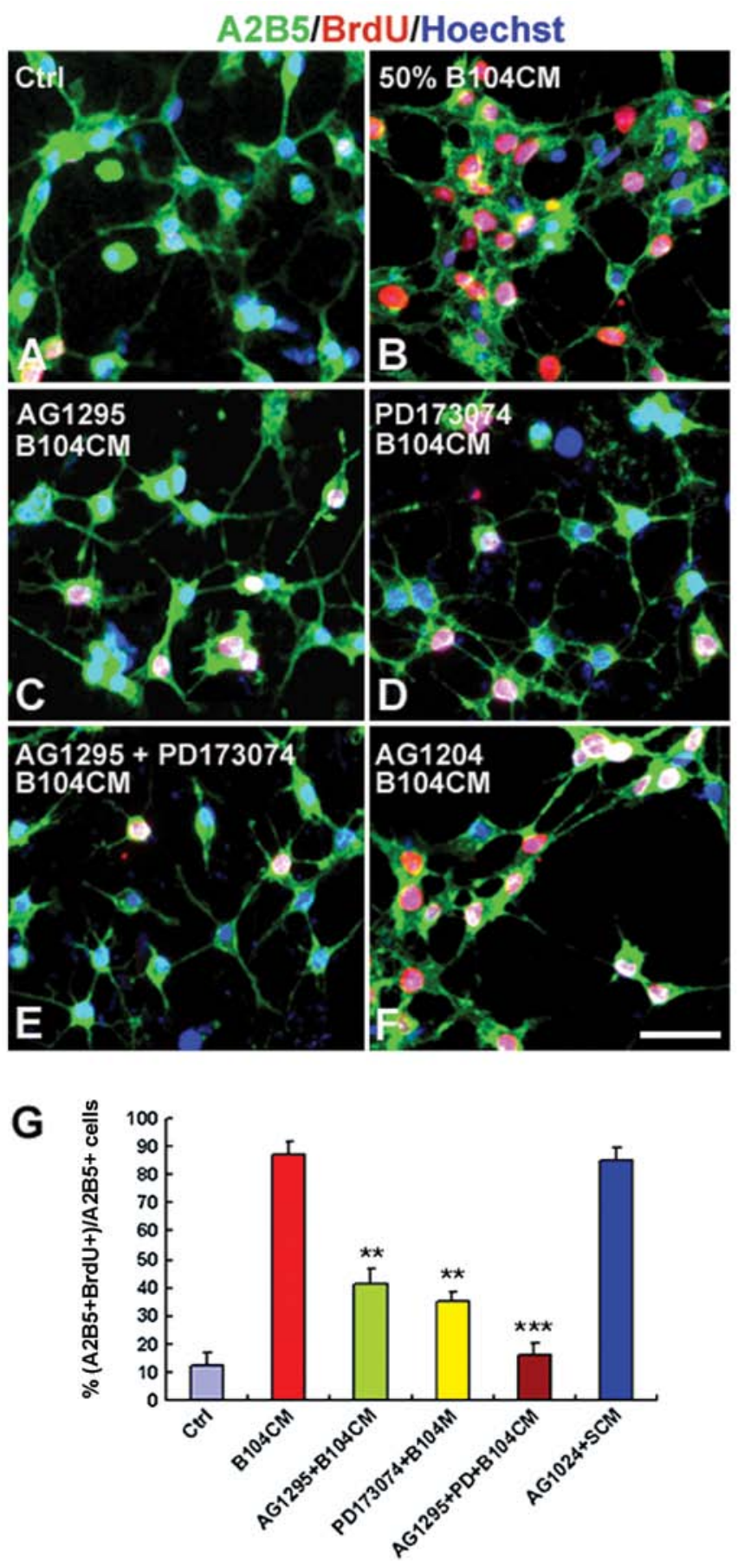

Figure 5. Effects of the growth factor inhibitors on B104CM-induced OPC proliferation. (A-F) Representative photomicrographs displayed the effects of pre-incubation of (C) AG1295, (D) PD173074, (E) AG1295 + PD173074 or (F) AG1204, with OPC cultures on OPC proliferation induced by B104CM using BrdU incorporation assay. (G) Quantification of percent BrdU-positive OPCs in the same groups ( $\mathrm{n}=5 /$ group) described in $(\mathrm{A}-\mathrm{F}){ }^{* *} \mathrm{P}<0.01,{ }^{* * *} \mathrm{P}<0.001$, compared to the control. Scale bar, $25 \mu \mathrm{m}$.

we performed immunofluorescence staining. The results showed that all 3 receptors, PDGFR, FGFR2 and IGF-1R, could be detected in OPCs (Fig. 4A-I). This finding, together with the result that these growth factors exist in B104CM, indicates that these three growth factors contained in B104CM may be factors which induce OPC proliferation.
PDGF-AA and bFGF are 2 key factors for B104CM-induced $O P C$ proliferation. To determine whether these three growth factors are key factors for B104CM-induced OPC proliferation, we next used AG1295 (a specific inhibitor of the PDGFR signal pathway), PD173074 (a specific inhibitor of the bFGFR signal pathway) and AG1204 (a specific inhibitor of the IGFR signal pathway) to observe whether OPC proliferation can be blocked or decreased following addition of these inhibitors. The result showed that administration of both AG1295 and PD173074 prior to the addition of B104CM decreased B104CM-induced OPC proliferation from $88.05 \pm 4.76 \%$ (Fig. $5 \mathrm{~B}$ ) to $41.62 \pm 5.29 \%$ (Fig. 5C and $\mathrm{G})(\mathrm{P}<0.01)$ and $35.25 \pm 3.28 \%$ (Fig. 5D and $\mathrm{G}$ ) $(\mathrm{P}<0.01)$, respectively. Moreover, the combination of AG1295 and PD173074 mostly blocked all proliferation of OPCs induced by B104CM (Fig. 5E and G) $(\mathrm{P}<0.001)$. However, we did not observe a significant effect of AG1204 on OPC proliferation (Fig. 5F and $\mathrm{G})(\mathrm{P}>0.05)$. These results suggest that PDGF-AA and bFGF in B104CM are 2 key factors that induce OPCs to proliferate.

\section{Discussion}

Previous reports have confirmed that B104CM can induce OPCs to expand when cultured in vitro (3), indicating that certain factors which exist in B104CM induce OPC proliferation. However, which factors within B104CM are key factors responsible for this induction has yet to be clarified. To understand the mechanism by which B104CM induces OPC proliferation, in the present study we investigated the possible factors involved in B104CM-induced OPC proliferation.

Firstly, we isolated and cultured E15 rat spinal cordderived OPCs and confirmed that almost all were A2B5- and NG2-postive cells and were therefore highly pure. The majority differentiated into oligodendrocytes in the absence of serum and nearly all differentiated into the type II astrocytes in the presence of $10 \%$ serum when both PDGF-AA and bFGF were withdrawn. These results demonstrated that these OPCs were characteristic. Next, we examined the effect of B104CM on the proliferation of OPCs using BrdU incorporation assay. Our results confirmed that $\mathrm{B} 104 \mathrm{CM}$ at several different concentrations significantly promoted proliferation of OPCs and reached their peak values in the presence of 50\% B104CM.

It has been well established that PDGF-AA and bFGF are important mitogens for the proliferation of OPCs $(2,12)$. Moreover, a previous study found that IGF-I also induces oligodendrocyte progenitor proliferation in vitro (6). These findings raise the possibility that PDGF-AA, bFGF and IGF-I could be potent candidates that mediate B104CM-induced OPC proliferation. To clarify this possibility, we firstly explored the mRNA expressions and protein contents of these 3 proteins in B104 cells and B104CM, respectively. As expected, we observed the mRNA expressions of all in B104 cells and their protein contents in B104CM prepared from B104 cells. The functions of growth factors rely on binding to their receptors (13). Our results demonstrated that OPCs expressed PDGFR, FGFR2 and IGF-IR, which further increases the possibility that these growth factors are key factors in B104CM-induced OPC proliferation.

To investigate whether these 3 growth factors mediate B104CM-induced OPC proliferation, we blocked the func- 
tions of these 3 factors by treatment of the specific inhibitors. AG1295 is a specific inhibitor of the PDGFR signal pathway (14-16). Our results showed that AG1295 markedly decreased OPC proliferation induced by B104CM. PD173074 is a selective inhibitor of the bFGFR signal pathway (17). We also confirmed that PD173074 significantly reduced OPC proliferation. Markedly, the combination of AG1295 and PD173074 mostly blocked all proliferation of B104CM-induced OPC proliferation, suggesting that PDGF-AA and bFGF in B104CM are key factors that induce OPCs to proliferate. However, we did not observe evident change following administration of AG1204, a specific inhibitor of the IGFR signal pathway (18), although it has been reported that IGF-I also induces oligodendrocyte progenitor proliferation in vitro (6). We consider the reason behind this may be a low concentration of IGF-I in B104CM, which is not enough to induce OPC proliferation. Although PDGF-AA and bFGF were confirmed to mediate B104CMinduced OPC proliferation, it must be noted that there may still be other components which are also involved in instructing OPC proliferation within B104CM.

In conclusion, the present study has provided convincing evidence to suggest that PDGF-AA and bFGF contained in $\mathrm{B} 104 \mathrm{CM}$ serve as the key inducing factors that instruct OPC proliferation in vitro. Identifying these molecules contributes to understanding the mechanism of B104CM-induced OPC proliferation.

\section{Acknowledgements}

This study was supported by the National Natural Science Foundation of China (nos. 81071268 and 81171465), the Science and Technological Fund of Anhui Province for Outstanding Youth (no. 10040606Y13), the Key Project of Chinese Ministry of Education (no. 210103), and a grant from the Advanced Programs of Anhui province academic and technical leader and candidates.

\section{References}

1. Pringle NP, Yu WP, Guthrie S, et al: Determination of neuroepithelial cell fate: induction of the oligodendrocyte lineage by ventral midline cells and sonic hedgehog. Dev Biol 177: 30-42, 1996.

2. Raff MC, Miller RH and Noble M: A glial progenitor cell that develops in vitro into an astrocyte or an oligodendrocyte depending on culture medium. Nature 303: 390-396, 1983.
3. Louis JC, Magal E, Muir D, et al: CG-4, a new bipotential glial cell line from rat brain, is capable of differentiating in vitro into either mature oligodendrocytes or type-2 astrocytes. J Neurosci Res 31: 193-204, 1992.

4. Noble M, Barnett SC, Bogler O, et al: Control of division and differentiation in oligodendrocyte-type-2 astrocyte progenitor cells. Ciba Found Symp 150: 227-243, 1990.

5. Tang DG, Tokumoto YM and Raff MC: Long-term culture of purified postnatal oligodendrocyte precursor cells. Evidence for an intrinsic maturation program that plays out over months. J Cell Biol 148: 971-984, 2000.

6. Cui QL and Almazan G: IGF-I-induced oligodendrocyte progenitor proliferation requires PI3K/Akt, MEK/ERK, and Src-like tyrosine kinases. J Neurochem 100: 1480-1493, 2007.

7. Mayer-Proschel M, Kalyani AJ, Mujtaba T and Rao MS: Isolation of lineage-restricted neuronal precursors from multipotent neuroepithelial stem cells. Neuron 19: 773-785, 1997.

8. Mujtaba T, Piper DR, Kalyani A, et al: Lineage-restricted neural precursors can be isolated from both the mouse neural tube and cultured ES cells. Dev Biol 214: 113-127, 1999.

9. Cao Q, Xu XM, Devries WH, et al: Functional recovery in traumatic spinal cord injury after transplantation of multineurotrophin-expressing glial-restricted precursor cells. J Neurosci 25: 6947-6957, 2005

10. Hu J, Deng L, Wang X and Xu XM: Effects of extracellular matrix molecules on the growth properties of oligodendrocyte progenitor cells in vitro. J Neurosci Res 87: 2854-2862, 2009.

11. Hu JG, Fu SL, Zhang KH, et al: Differential gene expression in neural stem cells and oligodendrocyte precursor cells: a cDNA microarray analysis. J Neurosci Res 78: 637-646, 2004.

12. Asakura K, Hunter SF and Rodriguez M: Effects of transforming growth factor-beta and platelet-derived growth factor on oligodendrocyte precursors: insights gained from a neuronal cell line. J Neurochem 68: 2281-2290, 1997.

13. McDermott U, Ames RY, Iafrate AJ, et al: Ligand-dependent platelet-derived growth factor receptor (PDGFR)-alpha activation sensitizes rare lung cancer and sarcoma cells to PDGFR kinase inhibitors. Cancer Res 69: 3937-3946, 2009.

14. Banai S, Wolf Y, Golomb G, et al: PDGF-receptor tyrosine kinase blocker AG1295 selectively attenuates smooth muscle cell growth in vitro and reduces neointimal formation after balloon angioplasty in swine. Circulation 97: 1960-1969, 1998.

15. Hu JG, Wang YX, Wang HJ, Bao MS, Wang Zh, Ge X, Wang FC, Zhou JS and Lü HZ: PDGF-AA mediates B104CM-induced oligodendrocyte precursor cell differentiation of embryonic neural stem cells through Erk, PI3K, and p38 signaling. J Mol Neurosci 46: 644-653, 2011.

16. He H, Levitzki A, Zhu HJ, et al: Platelet-derived growth factor requires epidermal growth factor receptor to activate p21-activated kinase family kinases. J Biol Chem 276: 2674126744, 2001.

17. Pardo OE, Latigo J, Jeffery RE, et al: The fibroblast growth factor receptor inhibitor PD173074 blocks small cell lung cancer growth in vitro and in vivo. Cancer Res 69: 8645-8651, 2009.

18. Luk F, Yu Y, Walsh WR and Yang JL: IGF1R-targeted therapy and its enhancement of doxorubicin chemosensitivity in human osteosarcoma cell lines. Cancer Invest 29: 521-532, 2011. 